\title{
The Fading Number and Degrees of Freedom in Non-Coherent MIMO Fading Channels: A Peace Pipe
}

\author{
Tobias Koch Amos Lapidoth \\ Swiss Federal Institute of Technology (ETH) Zurich
}

\begin{abstract}
New non-asymptotic upper bounds on the capacity of non-coherent multiple-input multiple-output (MIMO) Gaussian fading channels with memory are proposed. These upper bounds are used to derive upper bounds on the fading number of regular Gaussian fading channels and on the pre-log of nonregular ones. The resulting bounds are tight in the multiple-input single-output (MISO) spatially independent Gaussian case when the entries in the fading vector are either zero-mean or possess the same spectral distribution function.

A new approach is proposed for the derivation of lower bounds on the fading number of MIMO channels. This approach is applied to derive a lower bound on the fading number of spatially IID zero-mean Gaussian fading channels.

The new upper and lower bounds on the fading number demonstrate that when the number of receive antennas does not exceed the number of transmit antennas, the fading number of zero-mean spatially IID slowly varying Gaussian MIMO channels is proportional to the number of degrees of freedom, i.e., to the minimum of the number of transmit and receive antennas. We conjecture that the same is true also when the number of receive antennas exceeds the number of transmit antennas. The singleinput multiple-output case that was recently solved by Lapidoth \& Moser supports this conjecture.
\end{abstract}

\section{INTRODUCTION}

The subject of this paper is the capacity of multiple-input multiple-output (MIMO) discrete-time flat fading channels with memory. We present firm (non-asymptotic) upper bounds on channel capacity and also study its high signal-to-noise ratio (SNR) asymptotic behavior via new lower bounds on the fading number. We shall see that the notions of the fading number and the number of degrees of freedom are closely tied.

We begin with a description of the channel and with some definitions. We envision a channel with $n_{\mathrm{T}}$ transmit antennas and $n_{\mathrm{R}}$ receive antennas. Its time- $k(k \in \mathbb{Z})$ output $\mathbf{Y}_{k} \in \mathbb{C}^{n_{\mathrm{R}}}$ corresponding to the time- $k$ channel input $\mathbf{x}_{k} \in \mathbb{C}^{n_{\mathrm{T}}}$ is an $n_{\mathrm{R}}$-dimensional complex random vector that is given by

$$
\mathbf{Y}_{k}=\mathbb{H}_{k} \mathbf{x}_{k}+\mathbf{Z}_{k}
$$

where the random $n_{\mathrm{R}} \times n_{\mathrm{T}}$ complex matrix $\mathbb{H}_{k} \in \mathbb{C}^{n_{\mathrm{R}} \times n_{\mathrm{T}}}$ denotes the time- $k$ fading matrix and the random vector $\mathbf{Z}_{k} \in \mathbb{C}^{n_{\mathrm{R}}}$ denotes the additive noise. Here $\mathbb{Z}$ denotes the set of integers and $\mathbb{C}$ denotes the set of complex numbers.

These results were presented in part at the Winterschool on Coding and Information Theory, Bratislava, February 20-25, 2005. They will appear in [7].
We assume throughout that the vectors $\left\{\mathbf{Z}_{k}\right\}$ are independent and identically distributed (IID) according to a circularly symmetric complex multi-variate Gaussian law of zero mean and of covariance matrix $\sigma^{2} \cdot \mathrm{I}_{n_{\mathrm{R}}}$ with $\sigma>0$. Here, $\mathrm{I}_{n_{\mathrm{R}}}$ denotes the $n_{\mathrm{R}} \times n_{\mathrm{R}}$ identity matrix.

The matrix valued fading process $\left\{\mathbb{H}_{k}\right\}$ is assumed to be stationary \& ergodic and to satisfy the finite expected squared Frobenius norm condition

$$
\mathrm{E}\left[\left\|\mathbb{H}_{k}\right\|_{\mathrm{F}}^{2}\right]<\infty .
$$

The fading process $\left\{\mathbb{H}_{k}\right\}$ and the additive noise process $\left\{\mathbf{Z}_{k}\right\}$ are independent of each other and of a joint law that does not depend on the channel inputs $\left\{\mathbf{x}_{k}\right\}$.

The above conditions will be assumed throughout. Some theorems will require additional assumptions. These are defined next. We shall say that the fading process $\left\{\mathbb{H}_{k}\right\}$ is regular if it has a finite differential entropy rate, i.e., if

$$
h\left(\left\{\mathbb{H}_{k}\right\}\right) \triangleq \lim _{n \rightarrow \infty} \frac{1}{n} h\left(\mathbb{H}_{1}, \ldots, \mathbb{H}_{n}\right)>-\infty .
$$

Otherwise, we shall say that it is non-regular. We say that the fading process is Gaussian if $\left\{\mathbb{H}_{k}\right\}$ is a matrix-valued Gaussian process, i.e., if for any finite number of deterministic coefficients $\alpha_{r, t, k}$ the sum $\sum \alpha_{r, t, k} H_{k}(r, t)$ is a (complex) Gaussian random variable. Here and throughout $H_{k}(r, t)$ denotes the row- $r$ column- $t$ entry of the matrix $\mathbb{H}_{k}$, and it is implicitly assumed that $1 \leq r \leq n_{\mathrm{R}}$ and $1 \leq t \leq n_{\mathrm{T}}$. We say that the fading is spatially independent if the $n_{\mathrm{R}} \cdot n_{\mathrm{T}}$ processes $\left\{H_{k}(r, t)\right\}$ are independent. We say that the fading is spatially IID if they are additionally of the same law.

The capacity of this channel under an average power $\mathcal{E}_{\mathrm{s}}$ constraint is

$$
C_{\mathrm{Avg}}(\mathrm{SNR})=\lim _{n \rightarrow \infty} \frac{1}{n} \sup I\left(\mathbf{X}_{1}^{n} ; \mathbf{Y}_{1}^{n}\right)
$$

where $A_{\ell}^{m}$ stands for $A_{\ell}, \ldots, A_{m}$ and where the maximization is over all joint distributions on $\mathbf{X}_{1}^{n}$ that satisfy

$$
\frac{1}{n} \sum_{k=1}^{n} \mathrm{E}\left[\left\|\mathbf{X}_{k}\right\|^{2}\right] \leq \mathcal{E}_{\mathrm{s}} .
$$

(Here $\|\cdot\|$ denotes the Euclidean norm.) For the peak power A constrained capacity the maximization is over all joint distributions under which with probability one

$$
\left\|\mathbf{X}_{k}\right\| \leq \mathrm{A}, \quad 1 \leq k \leq n
$$


The SNR is defined depending on whether an average or peak power constraint is imposed: $\mathrm{SNR} \triangleq \mathcal{E}_{\mathrm{s}} / \sigma^{2}$ for an average constraint and $\mathrm{SNR} \triangleq \mathrm{A}^{2} / \sigma^{2}$ for a peak constraint.

For regular fading the fading number is defined by

$$
\chi\left(\left\{\mathbb{H}_{k}\right\}\right) \triangleq \varlimsup_{\mathrm{SNR} \rightarrow \infty}\{C(\mathrm{SNR})-\log \log \mathrm{SNR}\}
$$

where, unless it is clear from the context or if the distinction is immaterial, we add a subscript "Avg" to indicate that an average power constraint is imposed and add the subscript "PP" for peak power. Notice that for regular fading the fading numbers corresponding to both a peak power constraint and an average power constraint are finite [1]. Also, for any fading law, $\chi_{\mathrm{PP}}\left(\left\{\mathbb{H}_{k}\right\}\right) \leq \chi_{\text {Avg }}\left(\left\{\mathbb{H}_{k}\right\}\right)$.

The exact calculation of the fading number for (regular) fading channels with memory is a difficult task. An exact expression for the fading number for the single-input singleoutput (SISO) case $\left(n_{\mathrm{R}}=n_{\mathrm{T}}=1\right)$ is given in [1]:

$$
\chi\left(\left\{H_{k}\right\}\right)=\log \pi+\mathrm{E}\left[\log \left|H_{1}\right|^{2}\right]-h\left(\left\{H_{k}\right\}\right) .
$$

The single-input multiple-output (SIMO) $\left(n_{\mathrm{T}}=1\right)$ case was recently solved in [2]. Here we shall present results for the multiple-input single-output (MISO) $\left(n_{\mathrm{R}}=1\right)$ case when the fading is spatially independent and Gaussian. Specifically, Corollary 8 treats the case where the fading is spatially independent Gaussian with a zero mean vector, and Corollary 9 treats the case where $\left\{\mathbf{H}_{k}-\mathbf{E}\left[\mathbf{H}_{k}\right]\right\}$ is Gaussian and spatially IID. For MIMO channels we shall present lower bounds on the fading number, see Theorem $4 \& 5$, and an upper bound for Gaussian fading, see Theorem 6 \& Corollary 7.

For non-regular fading capacity can grow with the SNR in various ways [3], [4]. When it grows logarithmically in the SNR we define the pre-log under a peak power constraint by

$$
\Pi_{\mathrm{PP}} \triangleq \varlimsup_{\mathrm{SNR} \rightarrow \infty} \frac{C_{\mathrm{PP}}(\mathrm{SNR})}{\log \mathrm{SNR}}
$$

with an analogous definition for the pre-log $\Pi_{\mathrm{Avg}}$ under an average power constraint.

The pre-log for general MIMO fading channels is unknown. It was computed under a peak-power constraint for the SISO Gaussian case in [3], [4] where it was shown that $\Pi_{P P}$ is given by the Lebesgue measure of the set of harmonics in the interval $[-1 / 2,1 / 2]$ where the derivative of the spectral distribution function $(\mathrm{SDF})$ is zero:

$$
\Pi_{\mathrm{PP}}=\mu\left(\left\{\lambda: F^{\prime}(\lambda)=0\right\}\right)
$$

where $\mu$ denotes the Lebesgue measure and $F^{\prime}$ is the derivative of the SDF of the fading process.

Here we shall present an upper bound on $\Pi_{\mathrm{PP}}$ for spatially independent MIMO Gaussian fading in Corollaries 11 \& 12 and an exact expression for $\Pi_{\mathrm{PP}}$ for spatially independent MISO Gaussian fading in Corollary 13.

The rest of this paper is organized as follows. In Section II we describe new upper bounds on channel capacity under peak power constraints. In Section III we address the fading number. We present the new lower bounds, the new upper bounds, and the MISO cases where these bounds yield the exact fading number. Section IV deals with non-regular fading and the pre$\log$. It includes upper bounds on the pre-log for non-regular MIMO fading and the expression for the MISO case when the bounds are tight. In Section V we specialize our results to slowly varying Gauss-Markov MIMO fading so as to be able to relate our results to those of [5], [6]. We conclude with a discussion of the relationship between the fading number and degrees of freedom.

\section{UPPER BOUNDS ON CHANNEL CAPACITY}

In this section we extend the bounds of [4] and [3] to firm upper bounds on the capacity of MIMO fading channels.

Theorem 1 Consider a mean-D spatially independent Gaussian MIMO fading $\left\{\mathbb{H}_{k}\right\}$ such that the random process $\left\{H_{k}(r, t)-d(r, t)\right\}$ is a zero-mean unit-variance circularly symmetric complex Gaussian process with $S D F F_{r, t}(\cdot)$. Then

$$
\begin{aligned}
& C_{\mathrm{PP}}(\mathrm{SNR}) \\
& \leq C_{\mathrm{PP}, \mathrm{IID}}(\mathrm{SNR})+\max _{\|\hat{\mathbf{x}}\|=1} \sum_{r=1}^{n_{\mathrm{R}}} \log \frac{1+\delta^{2}}{\sum_{t=1}^{n_{\mathrm{T}}}|\hat{x}(t)|^{2} \epsilon_{r, t}^{2}\left(\delta^{2}\right)+\delta^{2}}
\end{aligned}
$$

where $\delta^{2}=1 / \mathrm{SNR}$ and where $C_{\mathrm{PP}, \mathrm{IID}}(\mathrm{SNR})$ denotes the capacity in the memoryless fading case. Here, $\epsilon_{r, t}^{2}\left(\delta^{2}\right)$ denotes the error in predicting the $(r, t)$-th component of the fading matrix from a noisy observation of its past, i.e.,

$$
\epsilon_{r, t}^{2}\left(\delta^{2}\right)=\exp \left\{\int_{-1 / 2}^{1 / 2} \log \left(F_{r, t}^{\prime}(\lambda)+\delta^{2}\right) \mathrm{d} \lambda\right\}-\delta^{2} .
$$

When the fading process $\left\{\mathbb{H}_{k}-E\left[\mathbb{H}_{k}\right]\right\}$ is spatially IID, we obtain from Theorem 1 the following corollary.

Corollary 2 Let the mean-D Gaussian MIMO fading $\left\{\mathbb{H}_{k}\right\}$ be such that the process $\left\{\mathbb{H}_{k}-\mathrm{D}\right\}$ is spatially IID with each component being a zero-mean unit-variance circularly symmetric complex Gaussian process of SDF $F(\cdot)$. Then, defining $\epsilon^{2}\left(\delta^{2}\right)=\epsilon_{r, t}^{2}\left(\delta^{2}\right)$, (11) becomes

$$
C_{\mathrm{PP}}(\mathrm{SNR}) \leq C_{\mathrm{PP}, \mathrm{IID}}(\mathrm{SNR})+n_{\mathrm{R}} \log \frac{1+1 / \mathrm{SNR}}{\epsilon^{2}(1 / \mathrm{SNR})+1 / \mathrm{SNR}}
$$

An upper bound on the capacity of spatially independent MISO fading channels can be found by using Theorem 1 with $n_{\mathrm{R}}=1$. The following theorem provides an upper bound that generalizes this bound to channels where the fading is not spatially independent.

For convenience we shall write the MISO fading process as a column vector and not as a row vector. Thus, the time- $k$ channel output $Y_{k}$ is given by

$$
Y_{k}=\mathbf{H}_{k}^{\top} \mathbf{x}_{k}+Z_{k}
$$

where $\mathbf{H}_{k}^{\top}$ denotes the transpose of $\mathbf{H}_{k}$. 
Theorem 3 Consider a mean-d Gaussian MISO fading channel where $\left\{\mathbf{H}_{k}-\mathbf{d}\right\}$ is a zero-mean circularly symmetric complex Gaussian process with matrix-valued SDF $\mathrm{F}(\cdot)$, i.e.,

$$
\mathrm{E}\left[\left(\mathbf{H}_{k+m}-\mathbf{d}\right)\left(\mathbf{H}_{k}-\mathbf{d}\right)^{\dagger}\right]=\int_{-1 / 2}^{1 / 2} e^{i 2 \pi m \lambda} \mathrm{dF}(\lambda)
$$

where $(\cdot)^{\dagger}$ denotes Hermitian conjugation. Assume further that the covariance matrix $\mathrm{K}=\mathrm{E}\left[\left(\mathbf{H}_{k}-\mathbf{d}\right)\left(\mathbf{H}_{k}-\mathbf{d}\right)^{\dagger}\right]$ is nonsingular. Then, the capacity is upper bounded by

$$
C_{\mathrm{PP}}(\mathrm{SNR}) \leq C_{\mathrm{PP}, \mathrm{IID}}(\mathrm{SNR})+\log \frac{\|\mathrm{K}\|}{\lambda_{\min }(1 / \mathrm{SNR})}
$$

where $\lambda_{\min }\left(\delta^{2}\right)$ denotes the smallest eigenvalue of the error covariance matrix $\Sigma\left(\delta^{2}\right)$ in predicting the present fading from a noisy observation of its past; and $\|\cdot\|$ denotes the Euclidean operator norm of matrices, i.e., the largest singular value.

An upper bound on the capacity of Gaussian SISO fading channels with memory can be found by using Theorem 1 with $n_{\mathrm{R}}=1$. For the case where the fading is additionally of zero mean, a non-asymptotic lower bound is given in [7].

Note that the results presented in this section hold for both regular and non-regular processes. For a detailed proof see [8].

\section{The FAding Number}

In this section we present upper and lower bounds on the fading number (7) of MIMO fading channels. We will assume throughout this section that all channels considered here satisfy the finite differential entropy rate condition (3).

\section{A. Lower Bounds}

Theorem 4 Consider a general (not necessarily Gaussian) stationary and ergodic fading process $\left\{\mathbb{H}_{k}\right\}$ satisfying the conditions (2) and (3). Let $\left\{\mathbb{H}_{k}\right\}$ be independent of the IID random vectors $\left\{\mathbf{X}_{k}\right\}$ taking value in $\mathbb{C}^{n_{\mathrm{T}}}$ and satisfying

$$
\mathrm{E}\left[\left\|\mathbf{X}_{k}\right\|^{2}\right]<\infty \text { and } \mathrm{E}\left[\log \left\|\mathbf{X}_{k}\right\|^{2}\right]>-\infty .
$$

Then the fading number $\chi_{\mathrm{Avg}}\left(\left\{\mathbb{H}_{k}\right\}\right)$ is lower bounded by

$$
\begin{aligned}
& \chi_{\operatorname{Avg}}\left(\left\{\mathbb{H}_{k}\right\}\right) \\
& \geq \chi\left(\left\{\mathbb{H}_{k} \mathbf{X}_{k}\right\}\right)+\lim _{\mathrm{n} \rightarrow \infty} \frac{1}{n} I\left(\left\{\mathbf{X}_{k}\right\}_{k=1}^{n} ;\left\{\mathbb{H}_{k} \mathbf{X}_{k}\right\}_{k=1}^{n}\right)
\end{aligned}
$$

where $\chi\left(\left\{\mathbb{H}_{k} \mathbf{X}_{k}\right\}\right)$ is the fading number of a SIMO channel with fading process $\left\{\mathbb{H}_{k} \mathbf{X}_{k}\right\}$. (For SIMO fading, peak-power and average power constraints yield the same fading number [2].)

Moreover, if the random variables $\left\{\mathbf{X}_{k}\right\}$ are bounded, then the lower bound (18) holds also for the fading number $\chi_{\mathrm{PP}}\left(\left\{\mathbb{H}_{k}\right\}\right)$ of the fading process $\left\{\mathbb{H}_{k}\right\}$ under a peak-power constraint.

Note that this theorem can be extended with some care to the case where the inputs $\left\{\mathbf{X}_{k}\right\}$ are block-wise IID, e.g., if $\left\{\left(\mathbf{X}_{2 k}, \mathbf{X}_{2 k+1}\right)\right\}$ are IID.

An exact expression for the fading number $\chi\left(\left\{\mathbb{H}_{k} \mathbf{X}_{k}\right\}\right)$ of the SIMO fading $\left\{\mathbb{H}_{k} \mathbf{X}_{k}\right\}$ is given in [2]. However, this expression is not easy to evaluate. It can always be lower bounded by considering linear combining at the receiver which reduces the SIMO channel to a SISO channel for which the fading number is easier to compute (8): $\chi\left(\left\{\mathbb{H}_{k} \mathbf{X}_{k}\right\}\right) \geq$ $\chi\left(\left\{\boldsymbol{\alpha}^{\top} \mathbb{H}_{k} \mathbf{X}_{k}\right\}\right)\left(\boldsymbol{\alpha} \in \mathbb{C}^{n_{\mathrm{R}}}\right.$ deterministic), or by ignoring the memory in $\left\{\mathbb{H}_{k} \mathbf{X}_{k}\right\}$, i.e., $\chi\left(\left\{\mathbb{H}_{k} \mathbf{X}_{k}\right\}\right) \geq \chi_{\mathrm{IID}}\left(\mathbb{H}_{1} \mathbf{X}_{1}\right)$, or by applying both reductions $\chi\left(\left\{\mathbb{H}_{k} \mathbf{X}_{k}\right\}\right) \geq \chi_{\mathrm{IID}}\left(\boldsymbol{\alpha}^{\top} \mathbb{H}_{1} \mathbf{X}_{1}\right)$ where the subscript "IID" denotes the fading number in the memoryless case with equal marginals. The advantage of the latter is that it only depends on the marginal law of $\left\{\mathbb{H}_{k}\right\}$.

A particular choice for $\left\{\mathbf{X}_{k}\right\}$ which we shall find useful is the zero-mean multi-variate isotropic Gaussian law. It greatly simplifies the analysis of the second term on the RHS of (18). With this choice, we can use Theorem 4 to establish the following result on slowly varying Gaussian fading channels.

Theorem 5 Let the MIMO fading $\left\{\mathbb{H}_{k}\right\}$ be spatially IID with each component of $\left\{\mathbb{H}_{k}\right\}$ being a circularly symmetric zeromean unit-variance complex Gaussian process of temporal autocorrelation function $K[\nu]=\mathrm{E}\left[H_{k+\nu}^{*}(r, t) H_{k}(r, t)\right], \quad \nu \in$ $\mathbb{Z}$, where $H^{*}$ denotes the complex conjugate of $H$. Let $n_{\min } \triangleq$ $\min \left\{n_{\mathrm{R}}, n_{\mathrm{T}}\right\}$ and let

$$
\begin{aligned}
\epsilon_{\max }^{2} & \triangleq \max _{1 \leq \nu \leq n_{\min +2}} \mathrm{E}\left[\left|H_{\nu}(r, t)-H_{0}(r, t)\right|^{2}\right] \\
& =2 \cdot \max _{1 \leq \nu \leq n_{\min +2}}(1-\operatorname{Re}\{K[\nu]\})
\end{aligned}
$$

where $\operatorname{Re}\{K[\nu]\}$ denotes the real part of $K[\nu]$. Then

$$
\chi_{\mathrm{Avg}}\left(\left\{\mathbb{H}_{k}\right\}\right) \geq n_{\min } \log \frac{1}{\epsilon_{\max }^{2}}+\operatorname{Const}\left(n_{\min }\right)
$$

where the correction term $\operatorname{Const}\left(n_{\min }\right)$ depends only on $n_{\min }$ and not on the autocorrelation $K[\cdot]$.

Note that by using Theorem 5 with ever more slightly truncated Gaussian laws one can show that the lower bound (20) also holds for a peak-power constraint. Further note that in the above theorem we can replace $\epsilon_{\max }^{2}$ with

$$
\tilde{\epsilon}_{\max }^{2}=2 \cdot \max _{1 \leq \nu \leq n_{\min +2}}(1-\operatorname{Re}\{K[\pi(\nu)]\})
$$

where $\pi(\cdot)$ is any permutation of the natural numbers $\mathbb{N}$.

\section{B. Upper Bounds}

Theorem 6 Consider a mean-D spatially independent Gaussian MIMO fading $\left\{\mathbb{H}_{k}\right\}$ such that the random process $\left\{H_{k}(r, t)-d(r, t)\right\}$ is a zero-mean unit-variance circularly symmetric complex Gaussian process with $S D F F_{r, t}(\cdot)$. Then

$$
\chi_{\text {Avg }}\left(\left\{\mathbb{H}_{k}\right\}\right) \leq \chi_{\text {Avg, IID }}\left(\mathbb{H}_{1}\right)+\max _{\|\hat{\mathbf{x}}\|=1} \sum_{r=1}^{n_{\mathrm{R}}} \log \frac{1}{\sum_{t=1}^{n_{\mathrm{T}}}|\hat{x}(t)|^{2} \epsilon_{r, t}^{2}}
$$

where $\epsilon_{r, t}^{2}$ is $\epsilon_{r, t}^{2}\left(\delta^{2}\right)$ of (12) evaluated at $\delta^{2}=0$.

From this theorem, we can derive an upper bound on the fading number when the fading process $\left\{\mathbb{H}_{k}-\mathrm{E}\left[\mathbb{H}_{k}\right]\right\}$ is spatially IID.

Corollary 7 Let the mean-D Gaussian MIMO fading $\left\{\mathbb{H}_{k}\right\}$ be such that the process $\left\{\mathbb{H}_{k}-\mathrm{D}\right\}$ is spatially IID with 
each component being a zero-mean unit-variance circularly symmetric complex Gaussian process of SDF $F(\cdot)$. Then, defining $\epsilon^{2}=\epsilon_{r, t}^{2}$, the bound (22) reads

$$
\chi_{\mathrm{Avg}}\left(\left\{\mathbb{H}_{k}\right\}\right) \leq \chi_{\mathrm{Avg}, \mathrm{IID}}\left(\mathbb{H}_{1}\right)+n_{\mathrm{R}} \log \frac{1}{\epsilon^{2}} .
$$

Note that $\chi_{\operatorname{Avg}, I I D}\left(\mathbb{H}_{1}\right)$ is unknown for general fading matrices $\mathbb{H}_{1}$. However, $\chi_{\text {Avg,IID }}\left(\mathbb{H}_{1}\right)$ is known in the case where $\mathbb{H}_{1}$ is rotation commutative, and an upper bound on $\chi_{\mathrm{IID}}\left(\mathbb{H}_{1}\right)$ is known when the $n_{\mathrm{R}} \times n_{\mathrm{T}}$ matrix $\mathbb{H}_{1}$ is of the form $\mathbb{H}_{1}=\mathrm{D}+\tilde{\mathbb{H}}_{1}$ where $D$ is deterministic and $\tilde{\mathbb{H}}_{1}$ is spatially IID with each component of $\tilde{\mathbb{H}}_{1}$ being a zero-mean unit-variance circularly symmetric complex Gaussian random variable (see [1]).

\section{MISO Fading}

An upper bound on the fading number of spatially independent Gaussian MISO channels follows from Theorem 6 by using that [1, Corollary 4.28]

$$
\chi_{\mathrm{Avg}, \mathrm{IID}}\left(\mathbf{H}_{1}\right)=\chi_{\mathrm{PP}, \mathrm{IID}}\left(\mathbf{H}_{1}\right)=-1+\log d_{*}^{2}-\operatorname{Ei}\left(-d_{*}^{2}\right)
$$

where $\operatorname{Ei}(-\cdot)$ denotes the exponential integral function and

$$
d_{*}=\max _{\|\hat{\mathbf{x}}\|=1} \frac{\left|\mathrm{E}\left[\mathbf{H}_{k}^{\top}\right] \hat{\mathbf{x}}\right|}{\sqrt{\operatorname{Var}\left(\mathbf{H}_{k}^{\top} \hat{\mathbf{x}}\right)}} .
$$

In the following results for MISO fading channels are given.

Corollary 8 Consider a regular zero-mean spatially independent Gaussian MISO flat fading channel where the SDF of the unit-variance process $\left\{H_{k}(t)\right\}$ is given by $F_{t}(\cdot)$. Then, irrespective of whether a peak or an average power constraint is imposed,

$$
\chi\left(\left\{\mathbf{H}_{k}\right\}\right)=-1-\gamma+\log \frac{1}{\epsilon_{\min }^{2}}
$$

where $\gamma \approx 0.577$ denotes Euler's constant and

$$
\epsilon_{\min }^{2}=\min _{1 \leq t \leq n_{\mathrm{T}}} \exp \left\{\int_{-1 / 2}^{1 / 2} \log F_{t}^{\prime}(\lambda) \mathrm{d} \lambda\right\} .
$$

Moreover, this fading number can be achieved with beam selection, i.e., by transmitting from the antenna that yields the smallest prediction error.

Corollary 9 Let the regular mean-d Gaussian MISO fading $\left\{\mathbf{H}_{k}\right\}$ be such that the process $\left\{\mathbf{H}_{k}-\mathbf{d}\right\}$ is spatially IID with each component being a zero-mean unit-variance circularly symmetric complex Gaussian process of SDF $F(\cdot)$. Then for both an average power constraint and a peak power constraint the fading number is

$$
\chi\left(\left\{\mathbf{H}_{k}\right\}\right)=-1+\log \|\mathbf{d}\|^{2}-\operatorname{Ei}\left(-\|\mathbf{d}\|^{2}\right)+\log \frac{1}{\epsilon^{2}} .
$$

Moreover, the fading number is achievable with beam forming.

The following theorem generalizes Theorem 6 to channels that are not spatially independent.
Theorem 10 Let the mean-d MISO fading process be such that the process $\left\{\mathbf{H}_{k}-\mathbf{d}\right\}$ is a zero-mean circularly symmetric complex Gaussian process with matrix-valued SDF $\mathrm{F}(\cdot)$, and with covariance matrix K. Furthermore, assume that the prediction error covariance matrix $\Sigma$ is non-singular. Then,

$$
\chi_{\text {Avg }}\left(\left\{\mathbf{H}_{k}\right\}\right) \leq-1+\log d_{*}^{2}-\operatorname{Ei}\left(-d_{*}^{2}\right)+\log \frac{\|\mathrm{K}\|}{\lambda_{\min }}
$$

with $d_{*}$ as in (25) and where $\lambda_{\min }$ is $\lambda_{\min }\left(\delta^{2}\right)$ of (16) evaluated at $\delta^{2}=0$.

\section{THE PRE-LOG}

In this section we shall extend the SISO results on the pre$\log [3],[4]$ to the multi-antenna case.

Corollary 11 Consider a mean-D spatially independent MIMO fading $\left\{\mathbb{H}_{k}\right\}$ where the random process $\left\{H_{k}(r, t)-\right.$ $d(r, t)\}$ is a zero-mean unit-variance circularly symmetric complex Gaussian process with SDF $F_{r, t}(\cdot)$. Then

$$
\Pi_{\mathrm{PP}} \leq \max _{1 \leq t \leq n_{\mathrm{T}}} \sum_{r=1}^{n_{\mathrm{R}}} \mu\left(\left\{\lambda: F_{r, t}^{\prime}(\lambda)=0\right\}\right) .
$$

Corollary 12 Consider a mean-D Gaussian MIMO fading $\left\{\mathbb{H}_{k}\right\}$ where $\left\{\mathbb{H}_{k}-\mathrm{D}\right\}$ is spatially IID with each component being a zero-mean unit-variance circularly symmetric complex Gaussian process of SDF $F(\cdot)$. Then

$$
\Pi_{\mathrm{PP}} \leq n_{\mathrm{R}} \cdot \mu\left(\left\{\lambda: F^{\prime}(\lambda)=0\right\}\right) .
$$

For spatially independent Gaussian MISO channels, the upper bound provided in Corollary 11 is tight.

Corollary 13 Consider a mean-d spatially independent Gaussian MISO fading $\left\{\mathbf{H}_{k}\right\}$ such that the process $\left\{H_{k}(t)-\right.$ $d(t)\}$ is a zero-mean unit-variance circularly symmetric complex Gaussian process of SDF $F_{t}(\cdot)$. Then, the pre-log is

$$
\Pi_{\mathrm{PP}}=\max _{1 \leq t \leq n_{\mathrm{T}}} \mu\left(\left\{\lambda: F_{t}^{\prime}(\lambda)=0\right\}\right) .
$$

Moreover, this pre-log can be achieved with beam selection.

\section{A Gauss-Markov FAding Process}

A very simple model for a slowly varying channel is the Gauss-Markov fading model [9], [5]. Here

$$
\mathbb{H}_{k}=\sqrt{1-\varepsilon^{2}} \mathbb{H}_{k-1}+\varepsilon \mathbb{W}_{k}
$$

where $\left\{\mathbb{W}_{k}\right\}$ is spatially IID with $\left\{W_{k}(r, t)\right\}$ consisting of IID zero-mean unit-variance circularly symmetric complex Gaussians. In the above $\varepsilon^{2}$ is the mean squared error of the one-step predictor of $H_{0}(r, t)$ from its infinite past.

In the following, we consider a MIMO Gauss-Markov fading channel with $n=n_{\mathrm{R}}=n_{\mathrm{T}}$ transmit and receive antennas. With the aid of Corollary 7 one can upper bound the fading number $\chi_{\operatorname{Avg}}\left(\left\{\mathbb{H}_{k}\right\}\right)$ as

$$
\chi_{\operatorname{Avg}}\left(\left\{\mathbb{H}_{k}\right\}\right) \leq n \log \frac{1}{\varepsilon^{2}}+K_{1}(n)
$$


where the correction term $\mathrm{K}_{1}(n)$ is a function of $n$ and not of $\varepsilon^{2}$. Similarly, we can use Theorem 5 to lower bound the fading number as

$$
\chi_{\text {Avg }}\left(\left\{\mathbb{H}_{k}\right\}\right) \geq n \log \frac{1}{\varepsilon^{2}}+K_{2}(n)+o\left(\varepsilon^{2}\right)
$$

where the $o\left(\varepsilon^{2}\right)$ term tends to zero as $\varepsilon^{2} \rightarrow 0$.

\section{The FAding Number And Degrees of Freedom}

The "number of degrees of freedom" $n_{\min }$ of a system employing $n_{\mathrm{T}}$ transmit antennas and $n_{\mathrm{R}}$ receive antennas is defined by $n_{\min } \triangleq \min \left\{n_{\mathrm{T}}, n_{\mathrm{R}}\right\}$. It plays an important role in the high-SNR asymptotic analysis of coherent MIMO fading channels [10] as well as in the asymptotic analysis of the block-constant fading model [11], [12].

The role of degrees of freedom in non-coherent communication is more subtle. Indeed, if the limit in (7) exists then the asymptotic expansion

$$
C(\mathrm{SNR})=\log (1+\log (1+\mathrm{SNR}))+\chi+o(1)
$$

indicates that at very high SNR, when the $\log \log$ SNR term dominates the fading number $\chi$, capacity grows doublelogarithmically in the SNR and the number of transmit and receive antennas hardly influences capacity.

Great care, however, must be exercised when applying this argument. For this argument to demonstrate the irrelevance of the degrees of freedom in determining channel capacity, the SNR must not only be large enough so that (36) be a good approximation, but it must also be large enough so that the $\log \log$ SNR term dominate the fading number $\chi$. While, as we shall argue, the approximation (36) begins to hold at reasonable SNRs, for the $\log \log$ SNR term to dominate the fading number $\chi$ the SNR must be larger than the double exponentiation of the fading number. When the fading number is large, as in slowly varying channels, this latter condition only begins to hold at extremely high SNRs (even though (36) begins to hold at relatively moderate SNRs).

What then is the role of degrees of freedom in slowly varying non-coherent communication? For slowly varying channels, degrees of freedom play a key role in determining the fading number! Indeed-at least when $n_{\mathrm{R}} \leq n_{\mathrm{T}}$-Theorem 5 \& Corollary 7 combine to prove that for very slowly varying fading channels the fading number is roughly proportional to $n_{\min }$.

The picture that emerges is thus the following. The approximation (36) is quite reasonable as of relatively moderate signal-to-noise ratios. For slowly varying channels the $\log \log$ SNR dominates the fading number only at extremely high SNRs. At these extremely high SNRs, degrees of freedom, indeed, hardly influence capacity. However, increasing the number of degrees of freedom increases the fading number $\chi$ and hence pushes this extremely high SNR regime further and further away. If we think of the fading number as an indication of the maximal rate at which power efficient communication is achievable on the channel, then we can say that for slowly varying spatially independent Gaussian fading this rate is roughly proportional to the number of degrees of freedom. Thus, increasing the number of degrees of freedom increases the practical limit on power-efficient communication over the channel.

The results of [5], [6] on slowly-varying Gauss-Markov channels are, in fact, in agreement with this picture. It is just critical to understand that when they write [5] "However, when the SNR gets much larger, the Lapidoth-Moser regime kicks in ..." they mean that it is only at much larger SNRs that the $\log \log$ SNR term in (36) dominates the fading number $\chi$. The applicability of (36) begins at far lower SNRs.

Note that our results on the fading number and degrees of freedom are not specific to Gauss-Markov fading. It suffices that the auto-correlation decay slowly and that the difference between the present fading and any fading in the past $n_{\min }+2$ symbols be of expected squared error that is not much larger than the prediction error based on the infinite past. That is, we require that

$$
\log \frac{\epsilon_{\max }^{2}}{\epsilon^{2}}
$$

be roughly a constant. (Here $\epsilon_{\max }$ is defined in (19) and $\epsilon^{2}$ is the prediction error in predicting the present value of the process $H_{k}(r, t)$ from its infinite past.) This is certainly the case for Gauss-Markov processes.

\section{ACKNOWLEDGMENT}

Discussions with S. Moser and H. Bölcskei are gratefully acknowledged.

\section{REFERENCES}

[1] A. Lapidoth and S. M. Moser, "Capacity bounds via duality with applications to multiple-antenna systems on flat fading channels," IEEE Trans. Inform. Theory, vol. 49, no. 10, pp. 2426-2467, Oct. 2003.

[2] _ - "The fading number of SIMO fading channels with memory," in Proc. IEEE Int. Symp. on Inf. Theory and its Appl., 2004, pp. 287-292.

[3] A. Lapidoth, "On the high SNR capacity of stationary Gaussian fading channels," in Proc. 41st Allerton Conf. Comm., Contr. and Comp., Monticello, Il, Oct. 1-3, 2003.

[4] — , "On the asymptotic capacity of stationary Gaussian fading channels," IEEE Trans. Inform. Theory, vol. 51, no. 2, pp. 437-446, Feb. 2005.

[5] R. Etkin and D. Tse, "Degrees of freedom in underspread MIMO fading channels," 2003, preprint.

[6] —_ "Degrees of freedom in underspread MIMO fading channels," in Proc. IEEE Int. Symp. on Inf. Theory, Yokohama, Japan, June $29-$ July 4, 2003.

[7] T. Koch and A. Lapidoth, "Degrees of freedom in non-coherent stationary MIMO fading channels," in Proc. Winterschool on Coding and Inform. Theory, Bratislava, Feb. 20-25, 2005, to appear.

[8] T. Koch, "On the asymptotic capacity of multiple-input single-output fading channels with memory," Master's thesis, Signal and Inform. Proc. Lab., ETH Zurich, Switzerland, Apr. 2004.

[9] R. R. Chen, B. Hajek, R. Koetter, and R. Madhow, "On fixed input distributions for noncoherent communication over high-SNR Rayleigh-fading channels," IEEE Trans. Inform. Theory, vol. 50, no. 12, pp. 3390-3396, Dec. 2004.

[10] İ. E. Telatar, "Capacity of multi-antenna Gaussian channels," Europ. Trans. Telecommun., vol. 10, no. 6, pp. 585-595, 1999.

[11] T. Marzetta and B. Hochwald, "Capacity of a multiple-antenna communication link in Rayleigh flat fading environment," IEEE Trans. Inform. Theory, vol. 45, pp. 139-157, Jan. 1999.

[12] L. Zheng and D. N. C. Tse, "Communicating on the Grassmann manifold: A geometric approach to the non-coherent multiple antenna channel," IEEE Trans. Inform. Theory, vol. 48, no. 2, pp. 359-383, Feb. 2002 . 